\title{
Stability of an additive functional equation in the spaces of generalized functions
}

Young-Su Lee

Correspondence: masuri@sogang. ac.kr

Department of mathematics, Sogang university, Seoul 121-741, Republic of Korea

\section{Abstract}

We reformulate the following additive functional equation with $n$-independent variables

$$
n f\left(\sum_{i=1}^{n} x_{i}\right)=\sum_{i=1}^{n} f\left(x_{i}\right)+\sum_{1 \leq i<j \leq n} f\left(x_{i}+x_{j}\right)
$$

as the equation for the spaces of generalized functions. Making use of the fundamental solution of the heat equation we solve the general solutions and the stability problems of this equation in the spaces of tempered distributions and Fourier hyperfunctions. Moreover, using the regularizing functions, we extend these results to the space of distributions.

2000 MSC: 39B82; 46F05.

Keywords: Cauchy functional equation, stability, distribution, heat kernel, Gauss transform

\section{Introduction}

A function $f: \mathbb{R} \rightarrow \mathbb{R}$ is called an additive function if and only if it satisfies the Cauchy functional equation

$$
f(x+y)=f(x)+f(y)
$$

for all $x, y \in \mathbb{R}$. It is well-known that every measurable solution of (1.1) is of the form $f(x)=a x$ for some constant $a$. In 1941, Hyers proved the stability theorem of (1.1) as follows:

Theorem 1.1 [1]. Let $E_{1}$ be a normed vector space, $E_{2}$ a Banach space. Suppose that $f$ $: E_{1} \rightarrow E_{2}$ satisfies the inequality

$$
\|f(x+y)-f(x)-f(y)\| \leq \varepsilon
$$

for all $x, y \in E_{1}$. Then, there exists the unique additive mapping $g: E_{1} \rightarrow E_{2}$ such that

$$
\|f(x)-g(x)\| \leq \varepsilon
$$

for all $x \in E_{1}$.

The above stability theorem was motivated by Ulam [2]. Forti [3] noticed that the theorem of Hyers is still true if $E_{1}$ is replaced by an arbitrary semigroup. In 1978, 
Rassias [4] generalized Hyers' result to the unbounded Cauchy difference. Thereafter, many authors studied the stability problems of (1.1) in various settings (see [5-7]).

During the last decades, stability problems of various functional equations have been extensively studied and generalized by a number of authors (see [8-13]). Among them, the following additive functional equation with $n$-independent variables:

$$
n f\left(\sum_{i=1}^{n} x_{i}\right)=\sum_{i=1}^{n} f\left(x_{i}\right)+\sum_{1 \leq i<j \leq n} f\left(x_{i}+x_{j}\right)
$$

was proposed by Nakmahachalasint [14], where $n$ is a positive integer with $n>1$. He proved that (1.2) is equivalent to (1.1). For that reason, we say that (1.2) is a generalization of the Cauchy functional equation. The stability theorem of (1.2) was also proved.

In this article, in a similar manner as in [15-19], we solve the general solutions and the stability problems of (1.2) in the spaces of generalized functions such as the space $\mathcal{S}^{\prime}\left(\mathbb{R}^{m}\right)$ of tempered distributions, the space $\mathcal{F}^{\prime}\left(\mathbb{R}^{m}\right)$ of Fourier hyperfunctions and the space $\mathcal{D}^{\prime}\left(\mathbb{R}^{m}\right)$ of distributions. Using the notions as in [15-19], we first reformulate (1.2) and the related inequality in the spaces of generalized functions as follows:

$$
\begin{aligned}
& n u \circ A=\sum_{i=1}^{n} u \circ P_{i}+\sum_{1 \leq i<j \leq n} u \circ B_{i j}, \\
& \left\|n u \circ A=\sum_{i=1}^{n} u \circ P_{i}-\sum_{1 \leq i<j \leq n} u \circ B_{i j},\right\| \leq \varepsilon,
\end{aligned}
$$

where $A, P_{i}$ and $B_{i j}$ are the functions defined by

$$
\begin{aligned}
& A\left(x_{1}, \ldots, x_{n}\right)=x_{1}+\cdots+x_{n} \\
& P_{i}\left(x_{1}, \ldots, x_{n}\right)=x_{i}, \quad 1 \leq i \leq n, \\
& B_{i j}\left(x_{1}, \ldots, x_{n}\right)=x_{i}+x_{j}, \quad 1 \leq i<j \leq n .
\end{aligned}
$$

Here, $\circ$ denotes the pullback of generalized functions and the inequality $\|v\| \leq \varepsilon$ in (1.4) means that $|\langle v, \varphi\rangle| \leq \varepsilon\|\varphi\|_{L^{1}}$ for all test functions $\phi$.

In Section 2, we prove that every solution $u$ in $\mathcal{F}^{\prime}\left(\mathbb{R}^{m}\right)$ or $\mathcal{S}^{\prime}\left(\mathbb{R}^{m}\right)$ of the equation (1.3) has the form

$$
u=a \cdot x,
$$

where $a \in \mathbb{C}^{m}$. Also, we prove that every solution $u$ in $\mathcal{S}^{\prime}\left(\mathbb{R}^{m}\right)$ or $\mathcal{F}^{\prime}\left(\mathbb{R}^{m}\right)$ of the inequality (1.4) can be written uniquely in the form

$$
u=a \cdot x+\mu(x)
$$

where $a \in \mathbb{C}^{m}$ and $\mu$ is a bounded measurable function such that $\| \mu||_{L^{\infty}} \leq \frac{2 \varepsilon}{n}$. Subsequently, in Section 3, these results are extended to the space $\mathcal{D}^{\prime}\left(\mathbb{R}^{m}\right)$.

\section{Stability in $\mathcal{F}^{\prime}$}

We first introduce the spaces of tempered distributions and Fourier hyperfunctions. Here, we use the $m$-dimensional notations, $|\alpha|=\alpha_{1}+\ldots+\alpha_{m}, \alpha !=\alpha_{1} ! \ldots \alpha_{m}$ !, 
$\alpha=\left(\alpha_{1}, \ldots, \alpha_{m}\right) \in \mathbb{N}_{0}^{m} \quad$ and $\quad \partial^{\alpha}=\partial_{1}^{\alpha_{1}} \ldots \partial_{m}^{\alpha_{m}}$, for $\zeta=\left(\zeta_{1}, \ldots, \zeta_{m}\right) \in \mathbb{R}^{m}$, $\alpha=\left(\alpha_{1}, \ldots, \alpha_{m}\right) \in \mathbb{N}_{0}^{m}$, where $\mathbb{N}_{0}$ is the set of non-negative integers and $\partial_{j}=\frac{\partial}{\partial \zeta_{j}}$.

Definition $2.1[20,21]$. We denote by $\mathcal{S}\left(\mathbb{R}^{m}\right)$ the Schwartz space of all infinitely differentiable functions $\phi$ in $\mathbb{R}^{m}$ satisfying

$$
\|\varphi\|_{\alpha, \beta}=\sup _{x \in \mathbb{R}^{m}}\left|x^{\alpha} \partial^{\beta} \varphi(x)\right|<\infty
$$

for all $\alpha, \beta \in \mathbb{N}_{0}^{m}$. A linear functional $u$ on $\mathcal{S}\left(\mathbb{R}^{m}\right)$ is said to be tempered distribution if there exists constant $C \geq 0$ and nonnegative integer $N$ such that

$$
|\langle u, \varphi\rangle| \leq C \sum_{|\alpha|,|\beta| \leq N} \sup _{x \in \mathbb{R}^{m}}\left|x^{\alpha} \partial^{\beta} \varphi\right|
$$

for all $\varphi \in \mathcal{S}\left(\mathbb{R}^{m}\right)$. The set of all tempered distributions is denoted by $\mathcal{S}^{\prime}\left(\mathbb{R}^{m}\right)$.

Note that tempered distributions are generalizations of $L^{p}$-functions. These are very useful for the study of Fourier transforms in generality, since all tempered distributions have a Fourier transform. Imposing the growth condition on $\|\cdot\|_{\alpha, \beta}$ in (2.1) a new space of test functions has emerged as follows:

Definition 2.2 [22]. We denote by $\mathcal{F}\left(\mathbb{R}^{m}\right)$ the set of all infinitely differentiable functions $\phi$ in $\mathbb{R}^{m}$ such that

$$
\| \varphi||_{A, B}=\sup _{x, \alpha, \beta} \frac{\left|x^{\alpha} \partial^{\beta} \varphi(x)\right|}{A^{|\alpha|} B^{|\beta|} \alpha ! \beta !}<\infty
$$

for some positive constants $A, B$ depending only on $\phi$. The strong dual of $\mathcal{F}\left(\mathbb{R}^{m}\right)$, denoted by $\mathcal{F}^{\prime}\left(\mathbb{R}^{m}\right)$, is called the Fourier hyperfunction.

It can be verified that the seminorm (2.2) is equivalent to

$$
\| \varphi||_{h, k}=\sup _{x, \alpha} \frac{\left|\partial^{\alpha} \varphi(x)\right| \exp k|x|}{h^{|\alpha|} \alpha !}<\infty
$$

for some constants $h, k>0$. It is easy to see the following topological inclusions:

$$
\mathcal{F}\left(\mathbb{R}^{m}\right) \hookrightarrow \mathcal{S}\left(\mathbb{R}^{m}\right), \quad \mathcal{S}^{\prime}\left(\mathbb{R}^{m}\right) \hookrightarrow \mathcal{F}^{\prime}\left(\mathbb{R}^{m}\right) .
$$

In order to solve the general solutions and the stability problems of (1.2) in the spaces $\mathcal{F}^{\prime}\left(\mathbb{R}^{m}\right)$ and $\mathcal{S}^{\prime}\left(\mathbb{R}^{m}\right)$ we employ the $m$-dimensional heat kernel, fundamental solution of the heat equation,

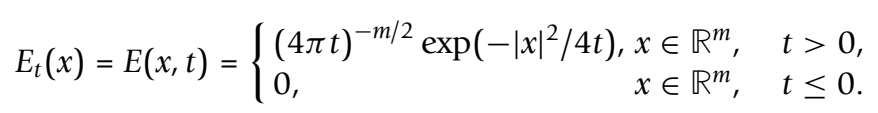

Since for each $t>0, E(\cdot, t)$ belongs to the space $\mathcal{F}\left(\mathbb{R}^{m}\right)$, the convolution

$$
\tilde{u}(x, t)=(u * E)(x, t)=\left\langle u_{y}, E_{t}(x-y)\right\rangle, \quad x \in \mathbb{R}^{m}, t>0
$$

is well-defined for all $u$ in $\mathcal{F}^{\prime}\left(\mathbb{R}^{m}\right)$, which is called the Gauss transform of $u$. Subsequently, the semigroup property

$$
\left(E_{t} * E_{s}\right)(x)=E_{t+s}(x), \quad x \in \mathbb{R}^{m}, \quad t, s>0
$$


of the heat kernel is very useful to convert Equation (1.3) into the classical functional equation defined on upper-half plane. We also use the following famous result, socalled heat kernel method, which states as follows:

Theorem 2.3 [23]. Let $u \in \mathcal{S}^{\prime}\left(\mathbb{R}^{m}\right)$. Then, its Gauss transform $\tilde{u}$ is a $C^{\infty}$-solution of the heat equation

$$
(\partial / \partial t-\Delta) \tilde{u}(x, t)=0
$$

satisfying

(i) There exist positive constants $C, M$ and $N$ such that

$$
|\tilde{u}(x, t)| \leq C t^{-M}(1+|x|)^{N} \text { in } \mathbb{R}^{m} \times(0, \delta) .
$$

(ii) $\tilde{u}(x, t) \rightarrow$ uas $t \rightarrow 0^{+}$in the sense that for every $\varphi \in \mathcal{S}\left(\mathbb{R}^{m}\right)$,

$$
\langle u, \varphi\rangle=\lim _{t \rightarrow 0^{+}} \int \tilde{u}(x, t) \varphi(x) d x .
$$

Conversely, every $C^{\infty}$-solution $U(x, t)$ of the heat equation satisfying the growth condition (2.4) can be uniquely expressed as $U(x, t)=\tilde{u}(x, t)$ for some $u \in \mathcal{S}^{\prime}\left(\mathbb{R}^{m}\right)$.

Similarly, we can represent Fourier hyperfunctions as a special case of the results as in [24]. In this case, the estimate (2.4) is replaced by the following:

For every $\varepsilon>0$, there exists a positive constant $C_{\varepsilon}$ such that

$$
|\tilde{u}(x, t)| \leq C_{\varepsilon} \exp (\varepsilon(|x|+1 / t)) \text { in } \mathbb{R}^{m} \times(0, \delta) .
$$

We are now going to solve the general solutions and the stability problems of (1.2) in the spaces of $\mathcal{F}^{\prime}\left(\mathbb{R}^{m}\right)$ and $\mathcal{S}^{\prime}\left(\mathbb{R}^{m}\right)$. Here, we need the following lemma.

Lemma 2.4. Suppose that $f: \mathbb{R}^{m} \times(0, \infty) \rightarrow \mathbb{C}$ is a continuous function satisfying

$$
n f\left(\sum_{i=1}^{n} x_{i}, \sum_{i=1}^{n} t_{i}\right)=\sum_{i=1}^{n} f\left(x_{i}, t_{i}\right)+\sum_{1 \leq i<j \leq n} f\left(x_{i}+x_{j}, t_{i}+t_{j}\right)
$$

for all $x_{1}, \ldots, x_{n} \in \mathbb{R}^{m}, t_{1}, \ldots, t_{n}>0$. Then, the solution $f$ has the form

$$
f(x, t)=a \cdot x+b t,
$$

where $a \in \mathbb{C}^{m}, b \in \mathbb{C}$.

Proof. Putting $\left(x_{1}, \ldots, x_{n}\right)=(0, \ldots, 0)$ in $(2.5)$ gives

$$
n f\left(0, \sum_{i=1}^{n} t_{i}\right)=\sum_{i=1}^{n} f\left(0, t_{i}\right)+\sum_{1 \leq i<j \leq n} f\left(0, t_{i}+t_{j}\right)
$$

for all $t_{1}, \ldots, t_{n}>0$. In view of (2.6), we see that

$$
c:=\limsup _{t \rightarrow 0^{+}} f(0, t)
$$

exists. Letting $t_{1}=\ldots=t_{n} \rightarrow 0^{+}$in (2.6) we have $c=0$. Replacing $\left(x_{3}, \ldots, x_{n}\right)=(0, \ldots$, 0 ) and letting $t_{3}=\ldots=t_{n} \rightarrow 0^{+}$in (2.5) yields

$$
f\left(x_{1}+x_{2}, t_{1}+t_{2}\right)=f\left(x_{1}, t_{1}\right)+f\left(x_{2}, t_{2}\right)
$$


for all $x_{1}, x_{2} \in \mathbb{R}^{m}, t_{1}, t_{2}>0$. Given the continuity, the solution $f$ of (2.7) has the form

$$
f(x, t)=a \cdot x+b t
$$

where $a \in \mathbb{C}^{m}, b \in \mathbb{C} . \square$

From the above lemma, we can find the general solutions of (1.2) in the spaces of $\mathcal{F}^{\prime}\left(\mathbb{R}^{m}\right)$ and $\mathcal{S}^{\prime}\left(\mathbb{R}^{m}\right)$. Taking the inclusions of (2.3) into account, it suffices to consider the space $\mathcal{F}^{\prime}\left(\mathbb{R}^{m}\right)$.

Theorem 2.5. Every solution $u$ in $\mathcal{F}^{\prime}\left(\mathbb{R}^{m}\right)$ (or $\mathcal{S}^{\prime}\left(\mathbb{R}^{m}\right)$, resp.) of the equation (1.3) has the form

$$
u=a \cdot x,
$$

where $a \in \mathbb{C}^{m}$.

Proof. Convolving the tensor product $E_{t_{1}}\left(x_{1}\right) \cdots E_{t_{n}}\left(x_{n}\right)$ of the heat kernels on both sides of (1.3) we have

$$
\begin{aligned}
& {[(u \circ A)}\left.*\left(E_{t_{1}}\left(x_{1}\right) \cdots E_{t_{n}}\left(x_{n}\right)\right)\right]\left(\xi_{1}, \ldots, \xi_{n}\right) \\
&=\left\langle u \circ A, E_{t_{1}}\left(\xi_{1}-x_{1}\right) \cdots E_{t_{n}}\left(\xi_{n}-x_{n}\right)\right\rangle \\
&=\left\langle u, \int \cdots \int E_{t_{1}}\left(\xi_{1}-x_{1}+x_{2}+\cdots+x_{n}\right) E_{t_{2}}\left(\xi_{2}-x_{2}\right) \cdots E_{t_{n}}\left(\xi_{n}-x_{n}\right) d x_{2} \cdots d x_{n}\right\rangle \\
&=\left\langle u, \int \cdots \int E_{t_{1}}\left(\xi_{1}+\cdots+\xi_{n}-x_{1}-\cdots-x_{n}\right) E_{t_{2}}\left(x_{2}\right) \cdots E_{t_{n}}\left(x_{n}\right) d x_{2} \cdots d x_{n}\right\rangle \\
&=\left\langle u,\left(E_{t_{1}} * \cdots * E_{t_{n}}\right)\left(\xi_{1}+\cdots+\xi_{n}-x_{1}\right)\right\rangle \\
&=\left\langle u, E_{t_{1}+\cdots+t_{n}}\left(\xi_{1}+\cdots+\xi_{n}\right)\right\rangle \\
&=\tilde{u}\left(\xi_{1}+\cdots+\xi_{n}, t_{1}+\cdots+t_{n}\right), \\
& {\left[\left(u \circ P_{i}\right)\right.}\left.*\left(E_{t_{1}}\left(x_{1}\right) \cdots E_{t_{n}}\left(x_{n}\right)\right)\right]\left(\xi_{1}, \ldots, \xi_{n}\right)=\tilde{u}\left(\xi_{i}, t_{i}\right), \\
& {\left[\left(u \circ B_{i j}\right) *\left(E_{t_{1}}\left(x_{1}\right) \cdots E_{t_{n}}\left(x_{n}\right)\right)\right]\left(\xi_{1}, \ldots, \xi_{n}\right)=\tilde{u}\left(\xi_{i}+\xi_{j}, t_{i}+t_{j}\right), }
\end{aligned}
$$

where $\tilde{u}$ is the Gauss transform of $u$. Thus, (1.3) is converted into the following classical functional equation:

$$
n \tilde{u}\left(\sum_{i=1}^{n} x_{i}, \sum_{i=1}^{n} t_{i}\right)=\sum_{i=1}^{n} \tilde{u}\left(x_{i}, t_{i}\right)+\sum_{1 \leq i<j \leq n} \tilde{u}\left(x_{i}+x_{j}, t_{i}+t_{j}\right)
$$

for all $x_{1}, \ldots, x_{n} \in \mathbb{R}^{m}, t_{1}, \ldots, t_{n}>0$. It follows from Lemma 2.4 that the solution $\tilde{u}$ of (2.8) has the form

$$
\tilde{u}(x, t)=a \cdot x+b t
$$

where $a \in \mathbb{C}^{m}, b \in \mathbb{C}$. Letting $t \rightarrow 0^{+}$in (2.9) we obtain

$$
u=a \cdot x .
$$

This completes the proof. $\square$

In what follows, we denote $B, P$ and $Q$ are the functions defined by

$$
B(x, y)=x+y, \quad P\left(x_{1}, x_{2}\right)=x_{1}, \quad Q\left(x_{1}, x_{2}\right)=x_{2}, \quad x, y \in \mathbb{R}^{m} .
$$

From the above theorem, we have the general solution of (1.1) in the spaces of $\mathcal{F}^{\prime}\left(\mathbb{R}^{m}\right)$ and $\mathcal{S}^{\prime}\left(\mathbb{R}^{m}\right)$ immediately. 
Corollary 2.6. Every solution $u$ in $\mathcal{F}^{\prime}\left(\mathbb{R}^{m}\right)\left(\right.$ or $\mathcal{S}^{\prime}\left(\mathbb{R}^{m}\right)$, resp.) of the equation

$$
u \circ B=u \circ P+u \circ Q
$$

has the form

$$
u=a \cdot x,
$$

where $a \in \mathbb{C}^{m}$.

We are going to prove the stability theorem of (1.2) in the spaces of $\mathcal{F}^{\prime}\left(\mathbb{R}^{m}\right)$ and $\mathcal{S}^{\prime}\left(\mathbb{R}^{m}\right)$ as follows:

Theorem 2.7. Suppose that $u$ in $\mathcal{F}^{\prime}\left(\mathbb{R}^{m}\right)\left(\right.$ or $\mathcal{S}^{\prime}\left(\mathbb{R}^{m}\right)$, resp.) satisfies the inequality (1.4). Then, there exists a unique $a \in \mathbb{C}^{m}$ such that

$$
\|u-a \cdot x\| \leq \frac{2 \varepsilon}{n} .
$$

Proof. Convolving the tensor product $E_{t_{1}}\left(x_{1}\right) \cdots E_{t_{n}}\left(x_{n}\right)$ of the heat kernels on both sides of (1.4) we have

$$
\left|n f\left(\sum_{i=1}^{n} x_{i}, \sum_{i=1}^{n} t_{i}\right)-\sum_{i=1}^{n} f\left(x_{i}, t_{i}\right)-\sum_{1 \leq i<j \leq n} f\left(x_{i}+x_{j}, t_{i}+t_{j}\right)\right| \leq \varepsilon
$$

for all $x_{1}, \ldots, x_{n} \in \mathbb{R}^{m}, t_{1}, \ldots, t_{n}>0$, where $f$ is the Gauss transform of $u$. Putting $\left(x_{1}\right.$, $\left.\ldots, x_{n}\right)=(0, \ldots, 0)$ in $(2.11)$ yields

$$
\left|n f\left(0, \sum_{i=1}^{n} t_{i}\right)-\sum_{i=1}^{n} f\left(0, t_{i}\right)-\sum_{1 \leq i<j \leq n} f\left(0, t_{i}+t_{j}\right)\right| \leq \varepsilon
$$

for all $t_{1}, \ldots, t_{n}>0$. In view of (2.12), we see that

$$
c:=\limsup _{t \rightarrow 0^{+}} f(0, t)
$$

exists. Letting $t_{1}=\ldots=t_{n} \rightarrow 0^{+}$in (2.12) gives

$$
|c| \leq \frac{2 \varepsilon}{n^{2}-n}
$$

Setting $\left(x_{3}, \ldots, x_{n}\right)=(0, \ldots, 0), t_{3}=\ldots=t_{n} \rightarrow 0^{+}$in $(2.11)$ and using (2.13) we obtain

$$
\left|f\left(x_{1}+x_{2}, t_{1}+t_{2}\right)-f\left(x_{1}, t_{1}\right)-f\left(x_{2}, t_{2}\right)\right| \leq \frac{2 \varepsilon}{n}
$$

for all $x_{1}, x_{2} \in \mathbb{R}^{m}, t_{1}, t_{2}>0$. Putting $\left(x_{1}, x_{2}\right)=(x, x),\left(t_{1}, t_{2}\right)=(t, t)$ in (2.14) and dividing the result by 2 we have

$$
\left|\frac{f(2 x, 2 t)}{2}-f(x, t)\right| \leq \frac{\varepsilon}{n}
$$

for all $x \in \mathbb{R}^{m}, t>0$. Making use of the induction argument yields

$$
\left|\frac{f\left(2^{k} x, 2^{k} t\right)}{2^{k}}-f(x, t)\right| \leq \frac{2 \varepsilon}{n}\left(1-\frac{1}{2^{k}}\right)
$$


for all $k \in \mathbb{N}, x \in \mathbb{R}^{m}, t>0$. Replacing $x, t$ by $2^{l} x, 2^{l} t$ in (2.15), respectively, and dividing the result by $2^{l}$ we can see that $2^{-k} f\left(2^{k} x, 2^{k} t\right)$ is a Cauchy sequence which converges uniformly. Now let

$$
A(x, t):=\lim _{k \rightarrow \infty} 2^{-k} f\left(2^{k} x, 2^{k} t\right)
$$

Then, we verify that $A(x, t)$ is the unique mapping in $\mathbb{R}^{m} \times(0, \infty)$ satisfying the equation

$$
n A\left(\sum_{i=1}^{n} x_{i}, \sum_{i=1}^{n} t_{i}\right)=\sum_{i=1}^{n} A\left(x_{i}, t_{i}\right)+\sum_{1 \leq i<j \leq n} A\left(x_{i}+x_{j}, t_{i}+t_{j}\right)
$$

for all $x_{1}, \ldots, x_{n} \in \mathbb{R}^{m}, t_{1}, \ldots, t_{n}>0$ and the inequality

$$
|f(x, t)-A(x, t)| \leq \frac{2 \varepsilon}{n}
$$

for all $x \in \mathbb{R}^{m}, t>0$. Given the continuity, the solution $A(x, t)$ of $(2.16)$ is of the form

$$
A(x, t)=a \cdot x+b t,
$$

where $a \in \mathbb{C}^{m}, b \in \mathbb{C}$. Letting $t \rightarrow 0^{+}$in (2.17) we obtain

$$
\|u-a \cdot x\| \leq \frac{2 \varepsilon}{n} .
$$

Now inequality (2.18) implies that $u-a \cdot x$ belongs to $\left(L^{1}\right)^{\prime}=L^{\infty}$. Thus, all the solution $u$ in $\mathcal{F}^{\prime}$ can be written uniquely in the form $u=a \cdot x+h(x)$, where $\| h||_{L^{\infty}} \leq \frac{2 \varepsilon}{n}$. $\square$

From the above theorem, we shall prove the stability theorem of (1.1) in the spaces of $\mathcal{F}^{\prime}\left(\mathbb{R}^{m}\right)$ and $\mathcal{S}^{\prime}\left(\mathbb{R}^{m}\right)$ as follows:

Corollary 2.8. Suppose that $u$ in $\mathcal{F}^{\prime}\left(\mathbb{R}^{m}\right)\left(\right.$ or $\mathcal{S}^{\prime}\left(\mathbb{R}^{m}\right)$, resp.) satisfies the inequality

$$
\|u \circ B-u \circ P-u \circ Q\| \leq \varepsilon .
$$

Then, there exists a unique $a \in \mathbb{C}^{m}$ such that

$$
\|u-a \cdot x\| \leq \varepsilon .
$$

\section{Stability in $\mathcal{D}^{\prime}$}

In this section, we shall extend the previous results to the space of distributions. Recall that a distribution $u$ is a linear functional on $C_{c}^{\infty}\left(\mathbb{R}^{m}\right)$ of infinitely differentiable functions on $\mathbb{R}^{m}$ with compact supports such that for every compact set $K \subset \mathbb{R}^{m}$ there exist constants $C>0$ and $N \in \mathbb{N}_{0}$ satisfying

$$
|\langle u, \varphi\rangle| \leq C \sum_{|\alpha| \leq N} \sup \left|\partial^{\alpha} \varphi\right|
$$


for all $\varphi \in C_{c}^{\infty}\left(\mathbb{R}^{m}\right)$ with supports contained in $K$. The set of all distributions is denoted by $\mathcal{D}^{\prime}\left(\mathbb{R}^{m}\right)$. It is well-known that the following topological inclusions hold:

$$
C_{c}^{\infty}\left(\mathbb{R}^{m}\right) \hookrightarrow \mathcal{S}\left(\mathbb{R}^{m}\right), \quad \mathcal{S}^{\prime}\left(\mathbb{R}^{m}\right) \hookrightarrow \mathcal{D}^{\prime}\left(\mathbb{R}^{m}\right) .
$$

As we seen in $[15,16,19]$, by the semigroup property of the heat kernel, Equation (1.3) can be controlled easily in the spaces $\mathcal{F}^{\prime}\left(\mathbb{R}^{m}\right)$ and $\mathcal{S}^{\prime}\left(\mathbb{R}^{m}\right)$. But we cannot employ the heat kernel in the space $\mathcal{D}^{\prime}\left(\mathbb{R}^{m}\right)$. Instead of the heat kernel, we use the function $\psi_{t}(x):=t^{-m} \psi\left(\frac{x}{t}\right), x \in \mathbb{R}^{m}, t>0$, where $\psi(x) \in C_{c}^{\infty}\left(\mathbb{R}^{m}\right)$ such that

$$
\psi(x) \geq 0, \quad \operatorname{supp} \psi(x) \subset\left\{x \in \mathbb{R}^{m}:|x| \leq 1\right\}, \quad \int \psi(x) d x=1 .
$$

For example, let

$$
\psi(x)= \begin{cases}A \exp \left(-\left(1-|x|^{2}\right)^{-1}\right), & |x|<1, \\ 0, & |x| \geq 1,\end{cases}
$$

where

$$
A=\left(\int_{|x|<1} \exp \left(-\left(1-|x|^{2}\right)^{-1}\right) d x\right)^{-1}
$$

then it is easy to see $\psi(x)$ is an infinitely differentiable function with support $\{x:|x|$ $\leq 1\}$. Usually, we call $\psi(x)$ to the regularizing function. If $u \in \mathcal{D}^{\prime}\left(\mathbb{R}^{m}\right)$, then for each $t$ $>0,\left(u^{*} \psi_{t}\right)(x)=\left\langle u_{y}, \psi_{t}(x-y)\right\rangle$ is a smooth function in $\mathbb{R}^{m}$ and $\left(u^{*} \psi_{t}\right)(x) \rightarrow u$ as $t \rightarrow$ $0^{+}$in the sense of distributions, that is, for every $\varphi \in C_{c}^{\infty}\left(\mathbb{R}^{m}\right)$

$$
\langle u, \varphi\rangle=\lim _{t \rightarrow 0^{+}} \int\left(u * \psi_{t}\right)(x) \varphi(x) d x
$$

Making use of the regularizing functions we can find the general solution of (1.2) in the space $\mathcal{D}^{\prime}\left(\mathbb{R}^{m}\right)$ as follows:

Theorem 3.1. Every solution $u$ in $\mathcal{D}^{\prime}\left(\mathbb{R}^{m}\right)$ of Equation (1.3) has the form

$$
u=a \cdot x,
$$

where $a \in \mathbb{C}^{m}$.

Proof. Convolving the tensor product $\psi_{t_{1}}\left(x_{1}\right) \cdots \psi_{t_{n}}\left(x_{n}\right)$ of the regularizing functions on both sides of (1.3) we have

$$
\begin{aligned}
& {[(u \circ A)}\left.*\left(\psi_{t_{1}}\left(x_{1}\right) \cdots \psi_{t_{n}}\left(x_{n}\right)\right)\right]\left(\xi_{1}, \ldots, \xi_{n}\right) \\
&=\left\langle u \circ A, \psi_{t_{1}}\left(\xi_{1}-x_{1}\right) \cdots \psi_{t_{n}}\left(\xi_{n}-x_{n}\right)\right\rangle \\
&=\left\langle u, \int \cdots \int \psi_{t_{1}}\left(\xi_{1}-x_{1}+x_{2}+\cdots+x_{n}\right) \psi_{t_{2}}\left(\xi_{2}-x_{2}\right) \cdots \psi_{t_{n}}\left(\xi_{n}-x_{n}\right) d x_{2} \cdots d x_{n}\right\rangle \\
&=\left\langle u, \int \cdots \int \psi_{t_{1}}\left(\xi_{1}+\cdots+\xi_{n}-x_{1}-\cdots-x_{n}\right) \psi_{t_{2}}\left(x_{2}\right) \cdots \psi_{t_{n}}\left(x_{n}\right) d x_{2} \cdots d x_{n}\right\rangle \\
&=\left\langle u,\left(\psi_{t_{1}} * \cdots * \psi_{t_{n}}\right)\left(\xi_{1}+\cdots+\xi_{n}-x_{1}\right)\right\rangle \\
&=\left(u * \psi_{t_{1}} * \cdots * \psi_{t_{n}}\right)\left(\xi_{1}+\cdots+\xi_{n}\right), \\
& {\left[\left(u \circ P_{i}\right)\right.}\left.*\left(\psi_{t_{1}}\left(x_{1}\right) \cdots \psi_{t_{n}}\left(x_{n}\right)\right)\right]\left(\xi_{1}, \ldots, \xi_{n}\right)=\left(u * \psi_{t_{i}}\right)\left(\xi_{i}\right), \\
& {\left[\left(u \circ B_{i j}\right) *\left(\psi_{t_{1}}\left(x_{1}\right) \cdots \psi_{t_{n}}\left(x_{n}\right)\right)\right]\left(\xi_{1}, \ldots, \xi_{n}\right)=\left(u * \psi_{t_{i}} * \psi_{t_{j}}\right)\left(\xi_{i}+\xi_{j}\right) . }
\end{aligned}
$$


Thus, (1.3) is converted into the following functional equation:

$$
\begin{aligned}
& n\left(u * \psi_{t_{1}} * \cdots * \psi_{t_{n}}\right)\left(x_{1}+\cdots+x_{n}\right) \\
& \quad=\sum_{i=1}^{n}\left(u * \psi_{t_{i}}\right)\left(x_{i}\right)+\sum_{1 \leq i<j \leq n}\left(u * \psi_{t_{i}} * \psi_{t_{j}}\right)\left(x_{i}+x_{j}\right)
\end{aligned}
$$

for all $x_{1}, \ldots, x_{n} \in \mathbb{R}^{m}, t_{1}, \ldots, t_{n}>0$. In view of (3.1), it is easy to see that

$$
f(x):=\limsup _{t \rightarrow 0^{+}}\left(u * \psi_{t}\right)(x)
$$

exists. Putting $\left(x_{1}, \ldots, x_{n}\right)=(0, \ldots, 0)$ and letting $t_{1}=\ldots=t_{n} \rightarrow 0^{+}$in $(3.1)$ yields $f(0)=$ 0 . Setting $\left(x_{1}, x_{2}, x_{3}, \ldots x_{n}\right)=(x, y, 0, \ldots, 0),\left(t_{1}, t_{2}\right)=(t, s)$ and letting $t_{3}=\ldots=t_{n} \rightarrow 0^{+}$ in (3.1) we have

$$
\left(u * \psi_{t} * \psi_{s}\right)(x+y)=\left(u * \psi_{t}\right)(x)+\left(u * \psi_{s}\right)(y)
$$

for all $x, y \in \mathbb{R}^{m}, t, s>0$. Letting $t \rightarrow 0^{+}$in (3.2) gives

$$
\left(u * \psi_{s}\right)(x+y)=f(x)+\left(u * \psi_{s}\right)(y)
$$

for all $x, y \in \mathbb{R}^{m}, s>0$. Putting $y=0$ in (3.3) yields

$$
f(x)=\left(u * \psi_{s}\right)(x)-\left(u * \psi_{s}\right)(0)
$$

for all $x \in \mathbb{R}^{m}, s>0$. Applying (3.4) to (3.3) we see that $f$ satisfies the Cauchy functional equation

$$
f(x+y)=f(x)+f(y)
$$

for all $x, y \in \mathbb{R}^{m}$. Since $f$ is a smooth function in view of (3.4), it follows that $f(x)=a$ $\cdot x$, where $a \in \mathbb{C}^{m}$. Thus, from (3.4), we have

$$
\left(u * \psi_{s}\right)(x)=a \cdot x+\left(u * \psi_{s}\right)(0) .
$$

Letting $s \rightarrow 0^{+}$in (3.5) we finally obtain

$$
u=a \cdot x .
$$

This completes the proof. $\square$

In a similar manner, we have the following corollary immediately.

Corollary 3.2. Every solution $u$ in $\mathcal{D}^{\prime}\left(\mathbb{R}^{m}\right)$ of Equation (2.10) has the form

$$
u=a \cdot x,
$$

where $a \in \mathbb{C}^{m}$.

Using the regularizing functions, Chung [17] extended the stability theorem of the Cauchy functional equation (1.1) to the space $\mathcal{D}^{\prime}\left(\mathbb{R}^{m}\right)$. Similarly, we shall extend the stability theorem of (1.2) mentioned in the previous section to the space $\mathcal{D}^{\prime}\left(\mathbb{R}^{m}\right)$.

Theorem 3.3. Suppose that $u$ in $\mathcal{D}^{\prime}\left(\mathbb{R}^{m}\right)$ satisfies the inequality (1.4). Then, there exists a unique $a \in \mathbb{C}^{m}$ such that

$$
\|u-a \cdot x\| \leq \frac{2 \varepsilon}{n} .
$$


Proof. It suffices to show that every distribution satisfying (1.4) belongs to the space $\mathcal{S}^{\prime}\left(\mathbb{R}^{m}\right)$. Convolving the tensor product $\psi_{t_{1}}\left(x_{1}\right) \cdots \psi_{t_{n}}\left(x_{n}\right)$ on both sides of (1.4) we have

$$
\begin{aligned}
& \mid n\left(u * \psi_{t_{1}} * \cdots * \psi_{t_{n}}\right)\left(x_{1}+\cdots+x_{n}\right) \\
& \quad-\sum_{i=1}^{n}\left(u * \psi_{t_{i}}\right)\left(x_{i}\right)-\sum_{1 \leq i<j \leq n}\left(u * \psi_{t_{i}} * \psi_{t_{j}}\right)\left(x_{i}+x_{j}\right) \mid \leq \epsilon
\end{aligned}
$$

for all $x_{1}, \ldots, x_{n} \in \mathbb{R}^{m}, t_{1}, \ldots, t_{n}>0$. In view of (3.6), it is easy to see that for each fixed $x$,

$$
f(x):=\limsup _{t \rightarrow 0^{+}}\left(u * \psi_{t}\right)(x)
$$

exists. Putting $\left(x_{1}, \ldots, x_{n}\right)=(0, \ldots, 0)$ and letting $t_{1}=\ldots=t_{n} \rightarrow 0^{+}$in (3.6) yields

$$
|f(0)| \leq \frac{2 \varepsilon}{n^{2}-n}
$$

Setting $\left(x_{1}, x_{2}, x_{3}, \ldots, x_{n}\right)=(x, y, 0, \ldots, 0),\left(t_{1}, t_{2}\right)=(t, s), t_{3}=\ldots=t_{n} \rightarrow 0^{+}$in (3.6), and using (3.7), we have

$$
\left|\left(u * \psi_{t} * \psi_{s}\right)(x+\gamma)-\left(u * \psi_{t}\right)(x)-\left(u * \psi_{s}\right)(\gamma)\right| \leq \frac{2 \varepsilon}{n}
$$

for all $x, y \in \mathbb{R}^{m}, t, s>0$. Putting $y=0$ in (3.8) we obtain

$$
\left|\left(u * \psi_{t} * \psi_{s}\right)(x)-\left(u * \psi_{t}\right)(x)-\left(u * \psi_{s}\right)(0)\right| \leq \frac{2 \varepsilon}{n}
$$

for all $x \in \mathbb{R}^{m}, t, s>0$. Letting $t \rightarrow 0^{+}$in (3.9) gives

$$
\left|\left(u * \psi_{s}\right)(x)-f(x)-\left(u * \psi_{s}\right)(0)\right| \leq \frac{2 \varepsilon}{n}
$$

for all $x \in \mathbb{R}^{m}, s>0$. From (3.8) and (3.10), we have

$$
|f(x+y)-f(x)-f(y)| \leq \frac{6 \varepsilon}{n}
$$

for all $x, y \in \mathbb{R}^{m}$. According to the result as in [1], there exists a unique function $g: \mathbb{R}^{m} \rightarrow \mathbb{C}$ satisfying the equation

$$
g(x+y)=g(x)+g(y)
$$

for all $x, y \in \mathbb{R}^{m}$ such that

$$
|f(x)-g(x)| \leq \frac{6 \varepsilon}{n}
$$

for all $x \in \mathbb{R}^{m}$. It follows from (3.10) and (3.11) that

$$
\left|\left(u * \psi_{s}\right)(x)-g(x)-\left(u * \psi_{s}\right)(0)\right| \leq \frac{12 \varepsilon}{n}
$$


for all $x \in \mathbb{R}^{m}, s>0$. Letting $s \rightarrow 0^{+}$in (3.12) we obtain

$$
\|u-g(x)\| \leq \frac{2(6 n-5) \varepsilon}{n^{2}-n} .
$$

Inequality (3.13) implies that $h(x):=u-g(x)$ belongs to $\left(L^{1}\right)^{\prime}=L^{\infty}$. Thus, we conclude that $u=g(x)+h(x) \in \mathcal{S}^{\prime}\left(\mathbb{R}^{m}\right)$.

From the above theorem, we have the following corollary.

Corollary 3.4. Suppose that $u$ in $\mathcal{D}^{\prime}\left(\mathbb{R}^{m}\right)$ satisfies the inequality (2.19). Then, there exists a unique $a \in \mathbb{C}^{m}$ such that

$$
\|u-a \cdot x\| \leq \varepsilon .
$$

\section{Competing interests}

The author declares that he has no competing interests.

Received: 24 July 2011 Accepted: 2 November 2011 Published: 2 November 2011

\section{References}

1. Hyers, DH: On the stability of the linear functional equation. Proc Nat Acad Sci USA. 27, 222-224 (1941). doi:10.1073/ pnas.27.4.222

2. Ulam, SM: Problems in Modern Mathematics. Wiley, New York (1964)

3. Forti, GL: The stability of homomorphisms and amenability, with applications to functional equations. Abh Math Sem Univ Hamburg. 57, 215-226 (1987). doi:10.1007/BF02941612

4. Rassias, ThM: On the stability of the linear mapping in Banach spaces. Proc Am Math Soc. 72, $297-300$ (1978). doi:10.1090/S0002-9939-1978-0507327-1

5. Gajda, Z: On stability of additive mappings. Int J Math Math Sci. 14, 431-434 (1991). doi:10.1155/S016117129100056X

6. Isac, G, Rassias, ThM: On the Hyers-Ulam stability of $\psi$-additive mappings. J Approx Theory. 72, 131-137 (1993). doi:10.1006/jath.1993.1010

7. Skof, F: On the approximation of locally $\delta$-additive mappings (Italian). Atti Accad Sci Torino Cl Sci Fis Mat Natur. 117 377-389 (1983)

8. Hyers, DH, Isac, G, Rassias, ThM: Stability of Functional Equations in Several Variables. Birkhäuser, Boston (1998)

9. Kannappan, Pl: Functional Equations and Inequalities with Applications. Springer, New York (2009)

10. Aczél, J, Dhombres, J: Functional Equations in Several Variables. Cambridge University Press, Cambridge (1989)

11. Czerwik, S: Functional Equations and Inequalities in Several Variables. World Scientific Publishing Co., Inc., River Edge (2002)

12. Găvruța, P: A generalization of the Hyers-Ulam-Rassias stability of approximately additive mappings. J Math Anal Appl. 184, 431-436 (1994). doi:10.1006/jmaa.1994.1211

13. Jun, K-W, Kim, H-M: On the stability of an n-dimensional quadratic and additive functional equation. Math Inequal Appl. 9, 153-165 (2006)

14. Nakmahachalasint, P: Hyers-Ulam-Rassias and Ulam-Gavruta-Rassias stabilities of an additive functional equation in several variables. Int J Math Math Sci 2007, 6 (2007). Art. ID 13437

15. Chung, J, Lee, S: Some functional equations in the spaces of generalized functions. Aequationes Math. $65,267-279$ (2003). doi:10.1007/s00010-003-2657-y

16. Chung, J, Chung, S-Y, Kim, D: The stability of Cauchy equations in the space of Schwartz distributions. J Math Anal Appl. 295, 107-114 (2004). doi:10.1016/j.jmaa.2004.03.009

17. Chung, J: A distributional version of functional equations and their stabilities. Nonlinear Anal. 62, 1037-1051 (2005) doi:10.1016/j.na.2005.04.016

18. Lee, Y-S, Chung, S-Y: The stability of a general quadratic functional equation in distributions. Publ Math Debrecen. $\mathbf{7 4}$ 293-306 (2009)

19. Lee, Y-S, Chung, S-Y: Stability of quartic functional equations in the spaces of generalized functions. Adv Difference Equ 2009, 16 (2009). Art. ID 838347

20. Hörmander, L: The Analysis of Linear Partial Differential Operators I. Springer, Berlin (1983)

21. Schwartz, L: Théorie des Distributions. Hermann, Paris (1966)

22. Chung, J, Chung, S-Y, Kim, D: A characterization for Fourier hyperfunctions. Publ Res Inst Math Sci. 30, $203-208$ (1994). doi:10.2977/prims/1195166129

23. Matsuzawa, T: A calculus approach to hyperfunctions III. Nagoya Math J. 118, 133-153 (1990)

24. Kim, KW, Chung, S-Y, Kim, D: Fourier hyperfunctions as the boundary values of smooth solutions of heat equations. Publ Res Inst Math Sci. 29, 289-300 (1993). doi:10.2977/prims/1195167274

doi:10.1186/1687-1847-2011-50

Cite this article as: Lee: Stability of an additive functional equation in the spaces of generalized functions.

Advances in Difference Equations 2011 2011:50. 